\title{
Golden Probe of Electroweak Symmetry Breaking
}

\author{
Yi Chen, ${ }^{1,2, *}$ Joe Lykken, ${ }^{3, \dagger}$ Maria Spiropulu, ${ }^{2, \ddagger}$ Daniel Stolarski, ${ }^{4, \S}$ and Roberto Vega-Morales ${ }^{5, \|}$ \\ ${ }^{1}$ CERN, European Organization for Nuclear Research, Geneva, Switzerland CH-1211 \\ ${ }^{2}$ Lauritsen Laboratory for High Energy Physics, California Institute of Technology, Pasadena, California 92115, USA \\ ${ }^{3}$ Theoretical Physics Department, Fermilab, P.O. Box 500, Batavia, Illinois 60510, USA \\ ${ }^{4}$ Ottawa-Carleton Institute for Physics, Carleton University, 1125 Colonel By Drive, Ottawa, Ontario K1S 5B6, Canada \\ ${ }^{5}$ Departamento de Física Teórica y del Cosmos and CAFPE, Universidad de Granada, \\ Campus de Fuentenueva, E-18071 Granada, Spain
}

(Received 5 September 2016; published 9 December 2016)

\begin{abstract}
The ratio of the Higgs couplings to $W W$ and $Z Z$ pairs, $\lambda_{W Z}$, is a fundamental parameter in electroweak symmetry breaking as well as a measure of the (approximate) custodial symmetry possessed by the gauge boson mass matrix. We show that Higgs decays to four leptons are sensitive, via tree level or one-loop interference effects, to both the magnitude and, in particular, overall sign of $\lambda_{W Z}$. Determining this sign requires interference effects, as it is nearly impossible to measure with rate information. Furthermore, simply determining the sign effectively establishes the custodial representation of the Higgs boson. We find that $h \rightarrow 4 \ell(4 \ell \equiv 2 e 2 \mu, 4 e, 4 \mu)$ decays have excellent prospects of directly establishing the overall sign at a high luminosity $13 \mathrm{TeV}$ LHC. We also examine the ultimate LHC sensitivity in $h \rightarrow 4 \ell$ to the magnitude of $\lambda_{W Z}$. Our results are independent of other measurements of the Higgs boson couplings and, in particular, largely free of assumptions about the top quark Yukawa couplings which also enter at one loop. This makes $h \rightarrow 4 \ell$ a unique and independent probe of electroweak symmetry breaking and custodial symmetry.
\end{abstract}

DOI: 10.1103/PhysRevLett.117.241801

Introduction.-The precise nature of electroweak symmetry breaking (EWSB) is one of the fundamental questions the LHC is poised to answer. Measurements of the recently discovered [1,2] Higgs-like boson at $125 \mathrm{GeV}$ are a critical component of this program, and the details of its interactions with the heavy gauge bosons, the $W$ and the $Z$, are of particular importance. Measurements of Higgs decays indicate that its couplings to $W W$ and $Z Z$ pairs are mediated at tree level via the operators,

$$
\mathcal{L}_{Z W} \supset \frac{h}{v}\left(g_{Z} m_{Z}^{2} Z^{\mu} Z_{\mu}+2 g_{W} m_{W}^{2} W^{\mu+} W_{\mu}^{-}\right) .
$$

The standard model (SM) predicts the values of $g_{Z}$ and $g_{W}$, but the SM need not give the full picture of EWSB. As we discuss below, there are viable alternative theories with exotic Higgs sectors that predict different values of these couplings and, in particular, the ratio $\lambda_{W Z}=g_{W} / g_{Z}$. This ratio, which can, in principle, be positive or negative, gives crucial information on the nature of EWSB and the electroweak properties of $h$.

While global fits to Higgs decay rates can probe the magnitude of $\lambda_{W Z}$ [3-6] in a fairly model independent manner, interference effects are needed to probe the overall

Published by the American Physical Society under the terms of the Creative Commons Attribution 3.0 License. Further distribution of this work must maintain attribution to the author(s) and the published article's title, journal citation, and DOI. sign. Combined measurements of the rare production channels $t H$ and $Z H$ may allow for sensitivity to the overall sign of $\lambda_{W Z}[6]$ through interference effects, but this requires a fit of the observables in multiple channels as well as various assumptions about the presence (or lack) of effects from physics beyond the SM. Finally, the rate of these processes is quite small and current data has very little sensitivity to the sign. It would thus be valuable to have an independent probe free of such considerations and that is directly sensitive to the overall sign of $\lambda_{W Z}$.

We propose to exploit tree level or one-loop interference effects in Higgs boson decays to four charged leptons (electrons and muons) as a new avenue for studying $\lambda_{W Z}$. These decays have been shown to be a powerful probe of the spin of the Higgs boson as well as the $C P$ and tensor structure of its effective couplings to gauge boson pairs [7-43]. The sensitivity to $\lambda_{W Z}$ arises from interference between the tree level $Z Z$ mediated process and one-loop amplitudes involving the $W$ boson which contribute to the effective $h V V$ couplings. Since the interference depends linearly on $\lambda_{W Z}$, this makes $h \rightarrow 4 \ell(4 \ell \equiv 2 e 2 \mu, 4 e, 4 \mu)$ decays sensitive to both the magnitude and, in particular, overall sign of $\lambda_{W Z}$. There is also typically a contribution from top quark loops. This has been utilized to study the $C P$ properties of the top quark Yukawa sector [44] by exploiting analogous one loop or tree level interference effects.

We also consider the assumption that the Higgs scalar potential respects the well-known "custodial" global $S U(2)$ symmetry [45] as implied by electroweak precision data [46]. In this case $\lambda_{W Z}$ depends only on the custodial representation 
[47] of $h$, which is restricted to be either a singlet as in the SM, or fiveplet as found in the Georgi-Machacek (GM) model [48-51] and its supersymmetric incarnation [52-54]. This allows only two possibilities [47] for the ratio:

$$
\lambda_{W Z}=+1 \text { (singlet) }, \quad \lambda_{W Z}=-1 / 2 \quad \text { (fiveplet) } .
$$

While there are loop-level custodial symmetry breaking corrections $[50,51,53]$ to $\lambda_{W Z}$ induced via hypercharge and Yukawa interactions, they are much too small to give the $\mathcal{O}(1)$ corrections needed to change the sign of the tree level predictions in Eq. (2). Therefore, a determination of the sign of $\lambda_{W Z}$ effectively establishes the custodial representation of the Higgs boson.

We comment that although current data favor $\left|\lambda_{W Z}\right|=1$, the central value is below one and $\left|\lambda_{W Z}\right|=1 / 2$ is still consistent at $\sim 3 \sigma[5,6]$. While a value of $\lambda_{W Z}=-1$ would indicate violation of custodial symmetry, this is a logical possibility. Furthermore, there exist models [53] where violation of custodial symmetry can occur while still predicting $\rho=1$ at tree level. Thus, directly establishing the overall sign is still important.

We utilize the $h \rightarrow 4 \ell$ framework developed in Refs. [19,26,28,36,38,40,44], which uses all kinematic observables in the normalized fully differential decay width to perform a matrix element analysis of the sensitivity to $\lambda_{W Z}$. Since we are only interested in a ratio of couplings, we can take advantage of this to use only shape information. Thus, because rate information is not used, our analysis is largely independent of the uncertainties and assumptions associated with Higgs production effects.

Under minimal assumptions about the top Yukawa sector, we estimate that a $13 \mathrm{TeV}$ LHC will become sensitive to the overall sign of $\lambda_{W Z}$ in $h \rightarrow 4 \ell$ decays with $\sim 1300$ signal events corresponding to $\mathcal{O}(800) \mathrm{fb}^{-1}$ of data assuming SM production and decay rates. We also estimate how much data is needed to distinguish between the two predictions of custodial symmetry [see Eq. (2)] as well as the ultimate LHC sensitivity in $h \rightarrow 4 \ell$ to $\left|\lambda_{W Z}\right|$. We find that $h \rightarrow 4 \ell$ decays should serve as a unique and complementary, but independent, probe of EWSB and custodial symmetry at the LHC and beyond.

Probing electroweak symmetry breaking at the LHC.We now examine various ways to probe $\lambda_{W Z}$ with $h \rightarrow 4 \ell$ decays at a $13 \mathrm{TeV}$ LHC. An in depth discussion of how $\lambda_{W Z}$ enters into the $h \rightarrow 4 \ell$ amplitude through the $h V V$ effective couplings, as well as details on how the top Yukawa sector and dominant $4 \ell$ background are incorporated into our analysis can be found in Refs. [40,44,55]. We will consider both "CMS-like" phase space cuts [56,57] and a set of "Loose" cuts as defined in Refs. [40,44], which greatly relax the requirement on the lepton pair invariant masses, allowing them to be as low as $4 \mathrm{GeV}$. More details on the discussion and statistical analysis procedures presented here can also be found in Refs. [19,26,28,36,38,40,44,55] for the interested reader.
Pinning down the sign of $\lambda_{W Z} \cdot-$ We begin by assessing how much data are needed to pin down the overall sign of $\lambda_{W Z}$. As discussed, this cannot be done with rate measurements alone and, under the assumption of custodial symmetry, effectively establishes the custodial representation of the Higgs boson.

Following Ref. [44] we construct the likelihood from the (normalized) signal and background fully differential cross sections. This likelihood is a function of the couplings $\left(g_{Z}, g_{W}\right)$ defined in Eq. (1) as well as $y_{t}$, which we treat as a nuisance parameter. The likelihood is built for each pseudo-data-set, and integrated over $y_{t}$ (or fixing it to its true value) to obtain the posterior likelihood as a function only of $\lambda_{W Z}$. When generating pseudo-data-sets, we consider two possibilities. The first is the SM as the true model, which predicts $\lambda_{W Z}=1$. In the second case we consider the other allowed possibility by custodial symmetry of $\lambda_{W Z}=-1 / 2$. An example of the posterior likelihood is shown in Fig. 1 for one pseudo-dataset containing $\mathcal{O}(2000)$ signal events assuming $\lambda_{W Z}=1$.

We treat the normalized posterior likelihood as a probability density of the extracted true value of $\lambda_{W Z}$. Given the observed pseudo-data-set, we obtain from the posterior likelihood a $p$ value that the true value of $\lambda_{W Z}$ is negative by taking the ratio of the area on the negative side of zero (shaded in turquoise in Fig. 1) to the total area. For each $p$ value we define a corresponding "effective" $\sigma$ by how much (number of standard deviations) of the tail we have to integrate a (normalized) Gaussian to get an equivalent area of $p$. This is repeated over many pseudo-data-sets giving a distribution

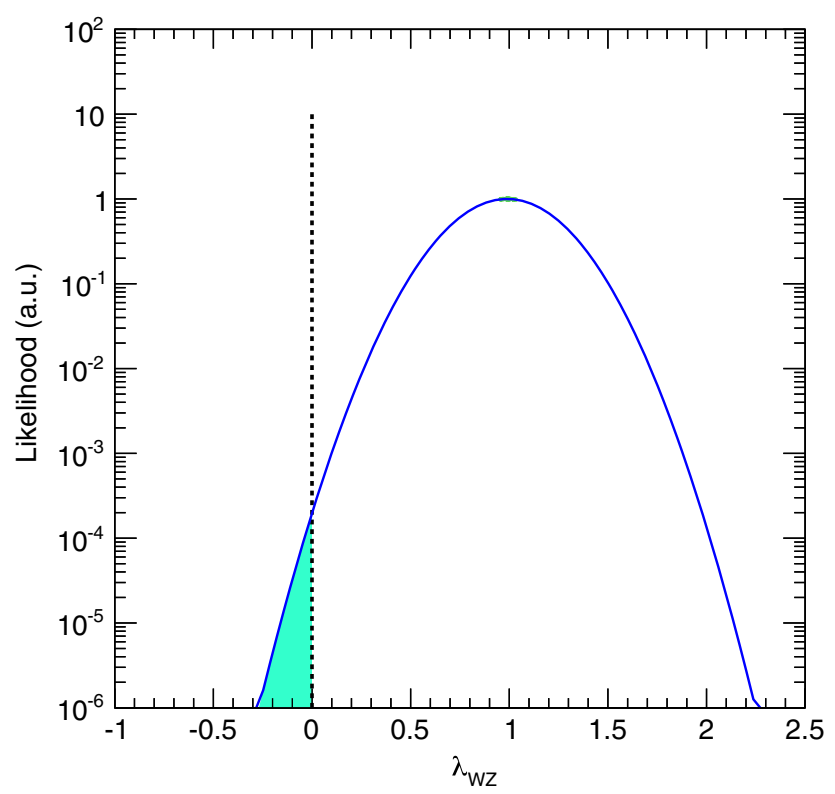

FIG. 1. Example of the posterior likelihood for one pseudodata-set containing $\mathcal{O}(2000)$ signal events and generated for the SM case with $\lambda_{W Z}=1$. The shaded turquoise region is translated into a probability (see Fig. 2) that the sign of $\lambda_{W Z}$ is negative. See text for more details. 


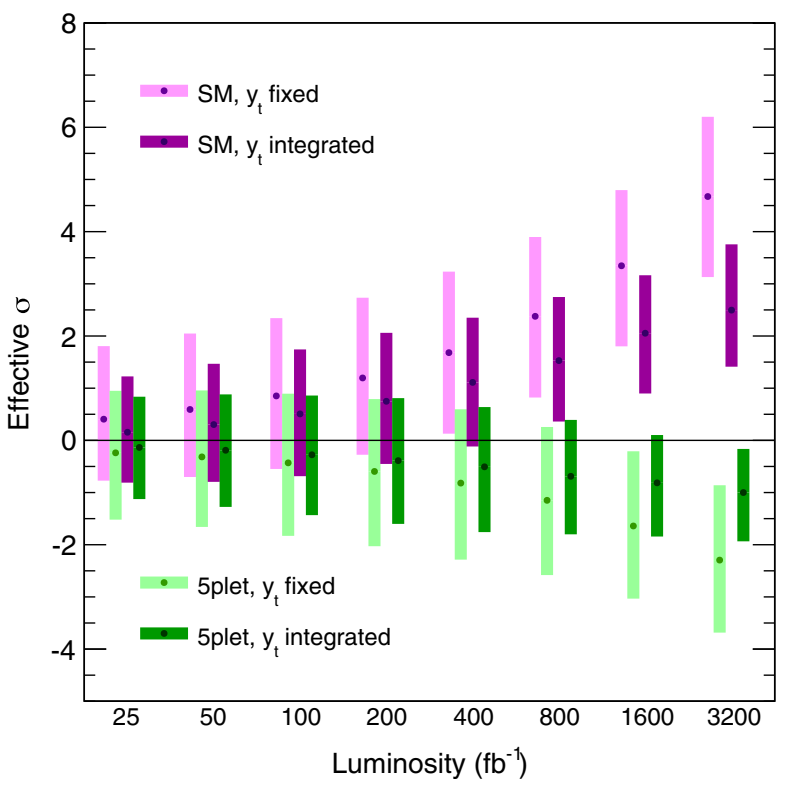

FIG. 2. Probability, in units of effective $\sigma$ 's, that the sign of $\lambda_{W Z}$ is negative as a function of luminosity. See text for more information.

of $\sigma$ 's which we interpret as the probability that the sign of $\lambda_{W Z}$ is negative.

In Fig. 2 we show the distribution of effective $\sigma$ 's for two separate cases. A negative value for the effective $\sigma$ indicates that the peak of the likelihood is on the negative side of $\lambda_{W Z}$, whereas a positive value represents a distribution centered on the positive side. The dots indicate the median value while the colored bands represent the central $68.3 \%$ interval of the distribution of effective $\sigma$ 's. In purple we show the SM while in green we show the case of a custodial fiveplet. For both cases we have considered two possibilities. The first (light shaded bands) is where $y_{t}$ is fixed to the true values, as predicted in the SM $\left(y_{t}=1\right)$ and for a custodial fiveplet $\left(y_{t}=0\right)$. In the second case (dark shaded bands), we assume only $\left|y_{t}\right| \lesssim 2$ via the use of a prior probability density function [58]. To interpret in terms of luminosity, we account for all relevant selection efficiencies [40,59] assuming SM production $(g g \rightarrow h$ plus VBF $[60,61])$ times branching ratio into $4 \ell$ [62].

We see in Fig. 2 that if the true model is the SM, a $13 \mathrm{TeV}$ LHC should begin pinning down the sign of $\lambda_{W Z}$ with $\mathcal{O}(800) \mathrm{fb}^{-1}$ of data, corresponding to $\mathcal{O}(1300)$ signal events for the more inclusive Loose cuts. While the sensitivity is degraded, whether we treat $y_{t}$ as a nuisance parameter which we integrate over or fix it to its true value, it does not qualitatively change this conclusion.

Of course, when fixing $y_{t}$ to its true value, we can establish the sign of $\lambda_{W Z}$ with more confidence. We see this in Fig. 2, where for the SM case an expected $\sigma \gtrsim 4$ can be obtained at very high LHC luminosities when $y_{t}$ is fixed, while only $\sigma \gtrsim 2$ is expected when integrated over. As constraints on the top Yukawa from direct measurements, such as $t$ th production $[5,64,65]$, increase in precision, stronger priors can be used leading to an increase in sensitivity to $\lambda_{W Z}$. We also see a stronger sensitivity to the SM case than to the fiveplet case due to the smaller value of $\left|\lambda_{W Z}\right|$ for the fiveplet.

We thus see that a high luminosity LHC has excellent prospects of establishing the overall sign of $\lambda_{W Z}$ in $h \rightarrow 4 \ell$ decays. As emphasized, under the assumption of custodial symmetry this also effectively establishes the custodial nature of the Higgs boson. Crucially, this does not rely on other measurements of Higgs boson couplings and is largely independent of the top Yukawa sector.

Testing custodial symmetry.-Motivated by considerations of custodial symmetry [47], we consider hypothesis testing to assess how much data is needed to discriminate between the two possibilities for $\lambda_{W Z}$ predicted by custodial symmetry in Eq. (2). Since custodial symmetry restricts the possibilities to two discrete choices, this makes it particularly suited to hypothesis testing.

We use the techniques developed in Ref. [18] and utilized in Refs. $[26,66,67]$ to construct a test statistic that measures the separation power between the two models allowed by custodial symmetry. This is done by constructing the likelihood ratio between the singlet and fiveplet hypotheses. Pseudoexperiments are conducted to obtain a distribution of these likelihood ratios, first assuming the SM as the "true" hypothesis and then repeated assuming the fiveplet is true. In each case, a distribution of likelihood ratios is obtained. The overlap (or lack thereof) between these two distributions is converted into a measure of the ability to discriminate between the two models. We follow closely the procedure in Ref. [26] with the additional step that we now include a nuisance parameter in $y_{t}$. We also present our results in terms of $p$ values instead of $\sigma$ 's.

In Fig. 3 we show the probability of mistaking a SM Higgs (custodial singlet) for a custodial fiveplet or vice versa as a function of data. Since there are small (subpercent) differences in selection efficiencies between the singlet and fiveplet, we plot the approximate number of signal events $\left(N_{S}\right.$ on bottom axis) to go along with the luminosity (top axis). We have again assumed SM production (gluon fusion plus VBF) times branching ratio and utilized the CMS-like phase space cuts (red) as well as the Loose phase space cuts (blue and green) discussed above and defined in Ref. [44]. In the green curve $\lambda_{W Z}$ and $y_{t}$ are fixed to their true values. In the red and blue curves $y_{t}$ is treated as a nuisance parameter and integrated over while imposing $\left|y_{t}\right| \lesssim 2$ and fixing $\lambda_{W Z}$ to its true value.

We see that $h \rightarrow 4 \ell$ decays should have the ability to discriminate between the two predictions of custodial symmetry for $\lambda_{W Z}$ at $95 \%$ confidence with $\mathcal{O}(3000)$ signal events corresponding to $\gtrsim 2000 \mathrm{fb}^{-1}$ of data. This conclusion is not greatly affected by whether we fix $y_{t}$ to its true value or treat it as a nuisance parameter. We also see that CMS-like cuts (red) are somewhat less sensitive than the more inclusive Loose cuts [40]. 


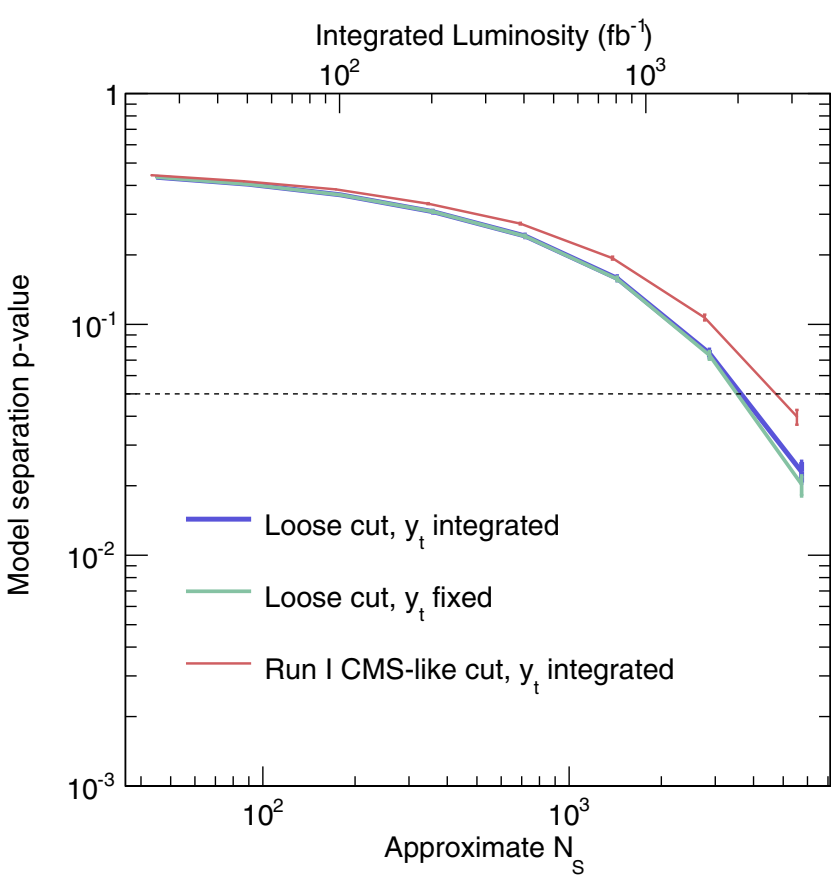

FIG. 3. Probability of mistaking a standard model Higgs boson (custodial singlet) for a custodial fiveplet or vice versa in terms of $p$ value. See text for more information.

Sensitivity to $\left|\lambda_{W Z}\right|$ at LHC and beyond.-Using the parameter extraction methods developed in Refs. $[36,38,40,44]$, we examine the sensitivity to $\left|\lambda_{W Z}\right|$. For this analysis we follow very closely the procedure based on a maximization of the likelihood which is described in Ref. [44], to which we refer the reader for more details.

We show in Fig. 4 curves for the average error, $\sigma\left(\lambda_{W Z}\right)$, defined in Refs. [36,39,40,44] as a function of the number of signal events (bottom axis) and luminosity $\times$ efficiency (top axis) assuming SM production ( $g g \rightarrow h$ plus VBF at $13 \mathrm{TeV}$ $[60,61])$. We also show a second luminosity axis assuming the nominal efficiency $(\sim 30 \%)$ for the Loose cuts. We fit to a true point of $\lambda_{W Z}=1$ again for both CMS-like (black) and Loose phase space cuts (blue, pink, and purple) [44]. We consider both $y_{t}$ as fixed (blue) and as a nuisance parameter for which we consider two separate cases. In the first we impose $\left|y_{t}\right| \lesssim 2$ (black and purple), but otherwise allow it to vary freely while in the second, no prior is applied (pink).

We see in Fig. 4 that $\mathcal{O}(1)$ precision on $\lambda_{W Z}$ may be achievable with $\sim 500-800$ signal events depending on whether $y_{t}$ is fixed or treated as a free nuisance parameter. Assuming $100 \%$ efficiency and SM production rates, this would require $\lesssim 100 \mathrm{fb}^{-1}$, though more realistically $\sim 300 \mathrm{fb}^{-1}$ is needed. We see, as expected, that once sufficient statistics are achieved, the sensitivity is much stronger when $y_{t}$ is fixed. As direct measurements of $y_{t}$ from $t$ th production $[5,64,65]$ become more precise, more restrictive priors on the top Yukawa can be used to enhance the sensitivity to $\lambda_{W Z}$ close to that achievable when fixing $y_{t}$. In this case, the ultimate LHC sensitivity could reach

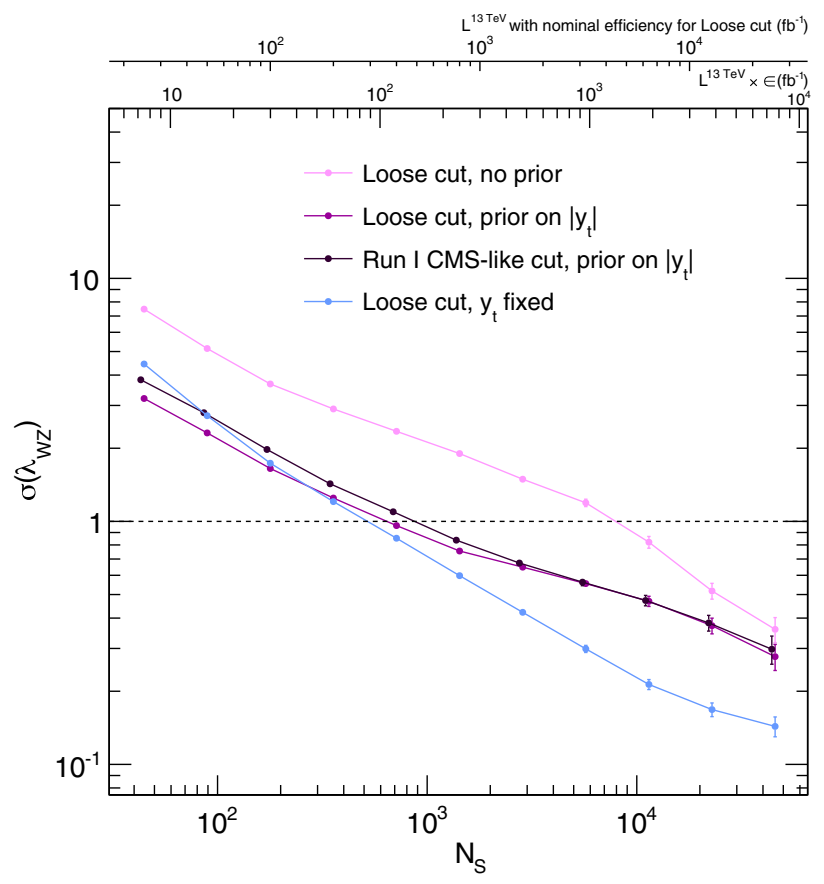

FIG. 4. $\sigma\left(\lambda_{W Z}\right)$ or average error (as defined in Refs. $[36,39,40,44])$ versus the number of signal events (bottom) and luminosity $\times$ efficiency (top). See text for more information.

$\mathcal{O}(20 \%-30 \%)$, again assuming $100 \%$ selection efficiencies and $\sim 3000 \mathrm{fb}^{-1}$.

Conclusions. - We have examined the possibility of using Higgs decays to four leptons to study the ratio of its couplings to $W W$ and $Z Z$ pairs, $\lambda_{W Z}$. This ratio is a crucial parameter of electroweak symmetry breaking and a probe of the wellknown custodial symmetry of the gauge boson mass matrix. Utilizing all observables in the normalized fully differential decay width, we construct a matrix element analysis to perform various statistical tests to assess the ability of $h \rightarrow 4 \ell$ decays to probe the magnitude and phase of $\lambda_{W Z}$.

In particular, we have emphasized that this channel is a uniquely effective probe of the sign of $\lambda_{W Z}$ and, furthermore, under the assumption of custodial symmetry, simply establishing the overall sign of $\lambda_{W Z}$ effectively determines the custodial representation of the Higgs boson. We have performed a likelihood shape analysis to estimate how much data will be needed to establish the sign at a $13 \mathrm{TeV}$ LHC. We find that $h \rightarrow 4 \ell$ decays will begin to become sensitive to the overall sign of $\lambda_{W Z}$ with as few as $\sim 1300$ signal events corresponding to $\mathcal{O}(800) \mathrm{fb}^{-1}$ of data assuming SM production and decay rates. As additional data is collected, the LHC should be able to firmly establish the overall sign, and therefore the custodial nature of the Higgs boson.

We have also performed hypothesis testing to assess the ability to discriminate between the two predictions of custodial symmetry for $\lambda_{W Z}$ in Eq. (2) and find they can be distinguished at $95 \%$ confidence with $~ 3000$ signal events corresponding to $\gtrsim 2000 \mathrm{fb}^{-1}$ of data, again assuming SM production and decay rates. In addition, we have examined 
the ultimate LHC sensitivity to $\left|\lambda_{W Z}\right|$ and estimate a precision of $\mathcal{O}(20-50) \%$ may be achievable by the end of the high luminosity LHC running.

Finally, we have emphasized the ability of $h \rightarrow 4 \ell$ decays to probe $\lambda_{W Z}$ without relying on other measurements of the Higgs boson couplings to electroweak vector bosons or the top quark. This makes $h \rightarrow 4 \ell$ deays a unique and independent probe of electroweak symmetry breaking at the LHC and beyond at future colliders.

We would like to thank Francisco del Aguila, Adam Falkowski, Heather Logan, Ian Low, Jose Santiago, and Martin Schmaltz for useful discussions. R. V.M. also thanks Fermilab and the Mainz Institute for Theoretical Physics (MITP) for their hospitality and partial support during the completion of this work as well as Tilman Plehn and the participants of the FIRSTRESULTS workshop for useful discussions. The work of Y. C. is supported by the Weston Havens Foundation and DOE Grant No. DE-FG0292-ER-40701. D. S. is supported in part by the Natural Sciences and Engineering Research Council of Canada (NSERC). R. V. M. is supported by MINECO, under Grant No. FPA2013-47836-C3-2-P.

*yi.chen@cern.ch

†lykken@fnal.gov

*stolar@physics.carleton.ca

§smaria@caltech.edu

"rvegamorales@ugr.es

[1] G. Aad et al. (ATLAS Collaboration), Phys. Lett. B 716, 1 (2012).

[2] S. Chatrchyan et al. (CMS Collaboration), Phys. Lett. B 716, 30 (2012).

[3] G. Aad et al. (ATLAS Collaboration), Phys. Lett. B 726, 120 (2013).

[4] S. Chatrchyan et al. (CMS Collaboration), Phys. Rev. D 89, 092007 (2014).

[5] T. Corbett, O. J. P. Eboli, D. Goncalves, J. Gonzalez-Fraile, T. Plehn, and M. Rauch, J. High Energy Phys. 08 (2015) 156.

[6] G. Aad et al. (ATLAS, CMS Collaborations), J. High Energy Phys. 08 (2016) 045.

[7] C. A. Nelson, Phys. Rev. D 37, 1220 (1988).

[8] A. Soni and R. M. Xu, Phys. Rev. D 48, 5259 (1993).

[9] D. Chang, W.-Y. Keung, and I. Phillips, Phys. Rev. D 48, 3225 (1993).

[10] V. D. Barger, K.-m. Cheung, A. Djouadi, B. A. Kniehl, and P. Zerwas, Phys. Rev. D 49, 79 (1994).

[11] T. Arens and L. Sehgal, Z. Phys. C 66, 89 (1995).

[12] S. Choi, D. J. Miller, M. Muhlleitner, and P. Zerwas, Phys. Lett. B 553, 61 (2003).

[13] C. Buszello, I. Fleck, P. Marquard, and J. van der Bij, Eur. Phys. J. C 32, 209 (2004).

[14] R. M. Godbole, D. J. Miller, and M. M. Muhlleitner, J. High Energy Phys. 12 (2007) 031.

[15] V. Kovalchuk, J. Exp. Theor. Phys. 107, 774 (2008).
[16] Q.-H. Cao, C. B. Jackson, W.-Y. Keung, I. Low, and J. Shu, Phys. Rev. D 81, 015010 (2010).

[17] Y. Gao, A. V. Gritsan, Z. Guo, K. Melnikov, M. Schulze, and N. V. Tran, Phys. Rev. D 81, 075022 (2010).

[18] A. De Rujula, J. Lykken, M. Pierini, C. Rogan, and M. Spiropulu, Phys. Rev. D 82, 013003 (2010).

[19] J. S. Gainer, K. Kumar, I. Low, and R. Vega-Morales, J. High Energy Phys. 11 (2011) 027.

[20] J. M. Campbell, W. T. Giele, and C. Williams, J. High Energy Phys. 11 (2012) 043.

[21] J. M. Campbell, W. T. Giele, and C. Williams, arXiv: 1205.3434.

[22] A. Belyaev, N. D. Christensen, and A. Pukhov, Comput. Phys. Commun. 184, 1729 (2013).

[23] B. Coleppa, K. Kumar, and H. E. Logan, Phys. Rev. D 86, 075022 (2012).

[24] S. Bolognesi, Y. Gao, A. V. Gritsan, K. Melnikov, M. Schulze, N. V. Tran, and A. Whitbeck, Phys. Rev. D 86, 095031 (2012).

[25] R. Boughezal, T. J. LeCompte, and F. Petriello, arXiv: 1208.4311.

[26] D. Stolarski and R. Vega-Morales, Phys. Rev. D 86, 117504 (2012).

[27] P. Avery, D. Bourilkov, M. Chen, T. Cheng, A. Drozdetskiy et al., Phys. Rev. D 87, 055006 (2013).

[28] Y. Chen, N. Tran, and R. Vega-Morales, J. High Energy Phys. 01 (2013) 182.

[29] T. Modak, D. Sahoo, R. Sinha, and H.-Y. Cheng, Phys. Rev. D 89, 068302 (2014).

[30] J. S. Gainer, J. Lykken, K. T. Matchev, S. Mrenna, and M. Park, Phys. Rev. Lett. 111, 041801 (2013).

[31] B. Grinstein, C. W. Murphy, and D. Pirtskhalava, J. High Energy Phys. 10 (2013) 077.

[32] Y. Sun, X.-F. Wang, and D.-N. Gao, Int. J. Mod. Phys. A 29, 1450086 (2014).

[33] I. Anderson et al., Phys. Rev. D 89, 035007 (2014).

[34] M. Chen, T. Cheng, J. S. Gainer, A. Korytov, K. T. Matchev, P. Milenovic, G. Mitselmakher, M. Park, A. Rinkevicius, and M. Snowball, Phys. Rev. D 89, 034002 (2014).

[35] G. Buchalla, O. Cata, and G. D’Ambrosio, Eur. Phys. J. C 74, 2798 (2014).

[36] Y. Chen and R. Vega-Morales, J. High Energy Phys. 04 (2014) 057.

[37] J. S. Gainer, J. Lykken, K. T. Matchev, S. Mrenna, and M. Park, Phys. Rev. D 91, 035011 (2015).

[38] Y. Chen, R. Harnik, and R. Vega-Morales, Phys. Rev. Lett. 113, 191801 (2014).

[39] Y. Chen, E. Di Marco, J. Lykken, M. Spiropulu, R. VegaMorales, and S. Xie, J. High Energy Phys. 01 (2015) 125.

[40] Y. Chen, R. Harnik, and R. Vega-Morales, J. High Energy Phys. 09 (2015) 185.

[41] B. Bhattacherjee, T. Modak, S. K. Patra, and R. Sinha, arXiv:1503.08924.

[42] M. Gonzalez-Alonso, A. Greljo, G. Isidori, and D. Marzocca, Eur. Phys. J. C 75, 341 (2015).

[43] A. Djouadi, J. Quevillon, and R. Vega-Morales, Phys. Lett. B 757, 412 (2016).

[44] Y. Chen, D. Stolarski, and R. Vega-Morales, Phys. Rev. D 92, 053003 (2015). 
[45] P. Sikivie, L. Susskind, M. B. Voloshin, and V. I. Zakharov, Nucl. Phys. B173, 189 (1980).

[46] K. A. Olive et al. (Particle Data Group), Chin. Phys. C 38, 090001 (2014).

[47] I. Low and J. Lykken, J. High Energy Phys. 10 (2010) 053.

[48] H. Georgi and M. Machacek, Nucl. Phys. B262, 463 (1985).

[49] M. S. Chanowitz and M. Golden, Phys. Lett. B 165, 105 (1985).

[50] J. F. Gunion, R. Vega, and J. Wudka, Phys. Rev. D 42, 1673 (1990).

[51] J. F. Gunion, R. Vega, and J. Wudka, Phys. Rev. D 43, 2322 (1991).

[52] L. Cort, M. Garcia, and M. Quiros, Phys. Rev. D 88, 075010 (2013).

[53] M. Garcia-Pepin, S. Gori, M. Quiros, R. Vega, R. VegaMorales, and T.-T. Yu, Phys. Rev. D 91, 015016 (2015).

[54] A. Delgado, M. Garcia-Pepin, and M. Quiros, J. High Energy Phys. 08 (2015) 159.

[55] Y. Chen, J. Lykken, M. Spiropulu, D. Stolarski, and R. Vega-Morales, arXiv:1608.02159 [Phys. Rev. D (to be published)].

[56] Tech. Rep. CMS-PAS-HIG-14-014, CERN, Geneva (2014).
[57] V. Khachatryan et al. (CMS Collaboration), Phys. Rev. D 92, 012004 (2015).

[58] Y. Chen, R. Vega-Morales et al. (to be published).

[59] Tech. Rep. CMS-PAS-HIG-15-004, CERN, Geneva (2016), URL https://cds.cern.ch/record/2139978.

[60] S. Dittmaier et al. (LHC Higgs Cross Section Working Group) arXiv:1101.0593.

[61] S. Heinemeyer et al. (LHC Higgs Cross Section Working Group) arXiv:1307.1347.

[62] The production rate for a fiveplet is a priori unknown since generically it does not couple to SM fermions and thus is not produced via gluon fusion through top quark loops. However, in models containing a fiveplet there can be additional colored states which could generate a coupling to gluons [52-54]. The VBF rate is also modified and there are nonSM production modes present [63].

[63] A. Delgado, M. Garcia-Pepin, M. Quiros, J. Santiago, and R. Vega-Morales, J. High Energy Phys. 06 (2016) 042.

[64] V. Khachatryan et al. (CMS Collaboration), Eur. Phys. J. C 74, 3076 (2014).

[65] V. Khachatryan et al. (CMS Collaboration), J. High Energy Phys. 09 (2014) 087.

[66] A. Falkowski and R. Vega-Morales, J. High Energy Phys. 12 (2014) 037.

[67] A. de la Puente and D. Stolarski, arXiv:1607.04276. 